\title{
Predictors of Attack Severity and Duration in Multiple Sclerosis: A Prospective Study
}

\author{
P. Naldi ${ }^{1}$, L. Collimedaglia ${ }^{1}$, D. Vecchio ${ }^{1}$, M.G. Rosso ${ }^{2}$, F. Perla ${ }^{2,3}$, A. Stecco ${ }^{4}$, F. Monaco ${ }^{1,3}$ and \\ M.A. Leone* ${ }^{* 1,3}$ \\ ${ }^{I}$ Clinica Neurologica, A. Avogadro University of Piemonte Orientale and A.O.U. Maggiore della Carità, Novara, Italy \\ ${ }^{2}$ SC Neurologia, S. Croce e Carle Hospital, Cuneo, Italy \\ ${ }^{3}$ Interdisciplinary Research Center of Autoimmune Diseases, Novara, Italy \\ ${ }^{4}$ Istituto di Radiologia Diagnostica e Interventistica, A.O.U. Maggiore della Carità, Novara, Italy
}

\begin{abstract}
Objective: To evaluate predictors of severity and duration of early Multiple Sclerosis (MS) attacks.
Methods: We analyzed 248 attacks in 95 patients in a prospective study. Severity: the difference between the EDSS score at the day of maximum worsening and the EDSS score before the onset of the attack. Duration: the time between the date of onset of the first symptom and the date of maximum improvement of the last symptom.

Results: The number of involved Functional Systems (FS), FS type (brainstem and pyramidal), and total attack duration were linked to severity. Number of FS involved, FS type (sphincteric and sensory), and severity of the attack were related to duration. Neither severity nor duration were correlated to other predictors: gender, age and season at attack onset, speed of onset, infections in the preceding month, age at first attack, season of birth and first attack, CSF examination, first brain MRI, recovery from the first attack. In the multivariate analysis, the Odds Ratio (OR) and Confidence Intervals (CI) for severe attacks was 3.6, 1.7-7.7 for involvement of pyramidal FS, 2.6, 1.2-6.0 for brainstem and 2.5, 1.2-5.3 for long attack duration. Sphincteric $(4.4 ; 1.7-11.0)$ and sensory FS $(1.8 ; 1.0-3.2)$ were the only variables explaining duration. The probability of a second moderate/severe or long attack was not influenced by severity or duration of the first.

Conclusions: FS are predictive of severity and duration of early MS attacks. Severity and duration of the first attack do not predict severity and duration of the second.
\end{abstract}

Keywords: Course, Duration, Multiple sclerosis, Prognosis, Recovery, Attack, Relapse, Severity.

\section{INTRODUCTION}

Clinical features of attacks in multiple sclerosis (MS) widely differ among patients and recovery is incomplete in one-third [1-3]. Incomplete recovery, indeed, is a strong predictor of a poor prognosis [4-7]. Severity has consistently been identified as the most powerful predictor of incomplete recovery, together with age and polysymptomatic onset $[1,2$, $4,8]$. However, factors that determine attack severity have been assessed in only one study [4] that identified younger age and brainstem/cerebellar location as predictors of severity, and showed that the severity of the first attack predicted that of the second. Factors that determine duration have never been evaluated. We have conducted an exploratory prospective study to look for factors that predict severity and duration of early attacks in patients with clinically isolated syndrome (CIS) and relapsing-remitting (RR) MS. We assumed that factors related to the characteristics of each attack (age and season at attack onset, previous infections, type, number, and speed of onset of symptoms, attack therapy) or to the characteristics of the patient or disease

*Address correspondence to this author at the Clinica Neurologica, "Maggiore della Carità" Hospital, Novara, C.so Mazzini, 18 - 28100 Novara, Italy; Tel: 0321/3733.218; Fax: 0321/3733.298;

E-mail: maurizio.leone@maggioreosp.novara.it course (gender, age at first attack of MS, season of birth and first attack, CSF and MRI features) influence severity and duration of MS attacks. In addition, we evaluated whether the severity and duration of and the recovery from the first MS attack are associated with the severity and duration of the second.

\section{MATERIALS AND METHODOLOGY}

\section{Patients and Follow-Up}

We enrolled 95 consecutive patients admitted to the Novara and Cuneo MS Centers from January 2001 (Cuneo from May 2002) to December 2003. Both centers are first-referral facilities which a total catchment area of 600,000 persons. We included patients presenting with CIS or RR-MS. CIS was defined as the occurrence of an acute or subacute event of the CNS affecting the optic nerves, brainstem or spinal cord of presumed inflammatory demyelinating origin in a patient with no history suggestive of an earlier demyelinating episode [9]. McDonald criteria [10] were used to diagnose MS. Primary progressive MS patients were excluded.

The cohort comprised 51 women and 44 men with a mean ages of 35.4 years (SD 10.6) and $31.4(9.2)(\mathrm{p}<0.05)$ at the first attack. The median interval between first symptom and visit was 51 days, (range 0-810). Patients were enrolled 
on their first visit to one of the two Centers. This corresponded to the first attack in 55 cases (58\%). The others were observed 1 to 27 months after the first attack (only 13 during or after the second). All patients had MRI; cerebrospinal fluid (CSF) was examined in 79. Other examinations were performed to rule out an alternative diagnosis. After the first visit, patients were clinically evaluated every 6 months until the end of the follow-up (July 31, 2005). During each visit, they were scored according to the Expanded Disability Status Scale (EDSS) [11]. Follow-up ranged from 19 to 55 months (mean 36.8; SD 10.2). At the end of follow-up, 21 were still CIS and 71 RR-MS; 3 patients had already entered secondary progression. For patients who presented a relapse in the last semester of the follow-up, an extra 12-month period (after July 31 ) was added to ascertain recovery (not considered in calculation of the mean follow-up).

Patients were instructed to phone the MS Center on the appearance of new symptoms and a visit was arranged in no more than 3 days. Further visits were planned after 3 and 5 days, and 1 month after the attack onset. Each attack was evaluated by an examiner neurologist blind to the treating neurologist. Each patient was provided at the beginning of the attack with a diary, derived from Liu [12] and asked to indicate each symptom and circle each day in which it was present. Diaries were collected at the end of the attack, and their information was integrated with that from the interview. Data on attacks not directly observed by us (anamnestic attacks, $\mathrm{N}=77,40$ first) were collected from hospital discharge letters, emergency departments and outpatient neurological reports, and integrated through interviews of patients and their relatives. Decision whether or not to treat an attack (either orally or intravenously) was left to the patient's physician. Seventeen patients were started on disease-modifying therapy (15 interferon, 2 glatiramer acetate) during the follow-up period, only in two cases before the second attack. The study was approved by the Ethical Committee of our Hospital; written informed consent was obtained to participate in the study and maintain data in the data-base.

\section{Attack Definition}

Attack was defined as "acute or subacute occurrence, recurrence or worsening of symptoms of neurologic dysfunction attributable to MS, lasting more than 24 hours after a period of at least 30 days of improvement or stability" [13]. Neurological deterioration of pre-existing symptoms accompanied by fever or concomitant infection (pseudoexcerbation) was not considered a attack. Paroxysmal episodes were considered as attacks when multiple and occurring over not less than 24 hours. Symptoms occurring within a month after the initial symptoms of an attack were considered to be part of the same episode [10]. For each attack, type of symptoms and signs and dates of occurrence were collected on an adhoc form, derived from Trojano et al [14]. Symptoms and signs were grouped to fit in with the Kurtzke Functional Systems (FS) [11]. For each symptom/sign we defined the date of onset, maximum worsening (zenith), start of improvement, and maximum improvement, defined as the date after which no further subjective improvement was noted. The score for each FS and the total EDSS score were calculated at onset, at the zenith and at the first examination after the day of maximum improvement. Agreement between the "at- tack" examiners was calculated with the Kappa statistics and varied from 0.7 to 0.9 for each FS [1].

\section{Definition of Severity, Duration and Predictive Factors}

Severity was graded according to the difference between the EDSS score at the day of maximum worsening and the EDSS score before the onset of the attack, as: very mild ( 0 or 1 point), mild ( 1.5 or 2 points), moderate ( 2.5 or 3 points), and severe (3.5 points or more). Duration was calculated as the time between the date of onset of the first symptom and the date of maximum improvement of the last symptom and classed as short ( $\leq 30$ days), intermediate (31-60 days) and long (>60 days). We evaluated two groups of predictive factors related to either the single attack or the single patient, including disease course characteristics. Attack-related factors were: age at the onset of the attack, season of the attack, type of FS involved, speed of onset, number of affected FS, infections in the preceding month, and therapy of the attack. Patient-related factors were: gender, age at first attack, season of birth and of first attack, oligoclonal bands at CSF examination, Link Index, CSF IgG value, total number and number of gadolinium-enhancing lesions at first brain MRI. Recovery after the first attack was considered incomplete when the difference between the score at the date of maximum improvement and the score before the onset of the attack was $\geq 1$ for at least one FS [1]. The definition of the other predictive variables are reported in our previous paper [1].

Data were analyzed with SAS [15]. Statistical comparisons were performed with Student's t test for independent samples, chi-square and chi-square for trend where appropriate. Spearman's coefficient was used for correlations. In view of the large number of comparisons, the Bonferroni correction for statistical significance was used. Odds Ratios (OR) with $95 \%$ confidence intervals (CI) were calculated. For multivariate analyses, possible predictors were dichotomized as follows: monosymptomatic vs. bi-polysymptomatic; severe vs. very mild+mild+moderate; long vs. intermediate+short, attack therapy vs. no therapy, season spring+summer vs. autumn+winter, age at attack onset $<30$ vs. $>=30$. A multiple logistic regression was performed using SAS/LOGISTIC [15], with duration and severity as the dependent variable, entering the predictor variables significant after univariate analysis and attack therapy (forward stepwise selection model with significance level for retention at 0.05).

In another statistical approach, we calculated the cumulative time-dependent probability of a second moderate/severe or long attack by the Kaplan-Meier method with $95 \%$ confidence intervals (CI). Date of entry was the date of onset of the first attack and the date of end-point was the date of onset of a severe/moderate or long attack, or July 31, 2005, whichever came first. Patients having a second nonmoderate/severe or non-long attack were censored at the time of their second attack. Categories of severity and duration were used as possible prognostic predictors of a attack. The log-rank test was used to test the significance of differences between groups. Multivariable Cox proportional hazard modeling was used to control for potential predictors of the probability of relapsing (SAS/PHREG) [15]. Predictors were entered into the model simultaneously. 


\section{RESULTS}

During the follow-up we counted 248 attacks (171 observed and 77 anamnestic): 95 first, 62 second, 33 third, 22 fourth, 16 fifth, and 20 subsequents. Very mild and mild attacks were more frequent $(\mathrm{p}<0.01)$ among the anamnestic $(46 / 77,60 \%)$ than the observed $(69 / 171,40 \%)$. Intravenous therapy was done for 26 anamnestic attacks (34\%), compared to 99 observed $(58 \%, \mathrm{p}<0.001)$. No significant differences were found between observed and anamnestic attacks in terms of frequency of all the other possible predictive factors; therefore anamnestic and observed attacks have been combined in the analysis.

Table 1. Severity of 248 MS Attacks, According to Attack-Related Characteristics

\begin{tabular}{|c|c|c|c|c|c|c|c|c|c|}
\hline \multicolumn{10}{|c|}{ Severity $^{a}$} \\
\hline \multirow[b]{2}{*}{ Predictive Factor } & \multicolumn{2}{|c|}{ very mild } & \multicolumn{2}{|c|}{ mild } & \multicolumn{2}{|c|}{ moderate } & \multicolumn{2}{|c|}{ severe } & \multirow[b]{2}{*}{ p-value ${ }^{b}$} \\
\hline & $\mathbf{N}$ & $\%$ & $\mathbf{N}$ & $\%$ & $\mathbf{N}$ & $\%$ & $\mathbf{N}$ & $\%$ & \\
\hline \multicolumn{10}{|c|}{ Number of FSs involved } \\
\hline Monosymptomatic & 52 & 34.9 & 60 & 40.3 & 29 & 19.5 & 8 & 5.4 & \\
\hline Bisymptomatic & 9 & 15.8 & 18 & 31.6 & 19 & 33.3 & 11 & 19.3 & $<0.0001^{\mathrm{c}}$ \\
\hline Polysymptomatic & 4 & 9.5 & 11 & 26.2 & 11 & 26.2 & 16 & 38.1 & \\
\hline \multicolumn{10}{|c|}{$\underline{\text { Type of FS involved }}^{\mathrm{d}}$} \\
\hline Pyramidal yes & 18 & 18.4 & 33 & 33.7 & 24 & 24.5 & 23 & 23.5 & $=0.003$ \\
\hline no & 47 & 31.3 & 56 & 37.3 & 35 & 23.3 & 12 & 8.0 & \\
\hline Cerebellar yes & 6 & 18.8 & 7 & 21.9 & 9 & 28.1 & 10 & 31.3 & n.s \\
\hline no & 59 & 27.3 & 82 & 38.0 & 50 & 23.2 & 25 & 11.6 & \\
\hline Brainstem yes & 5 & 9.8 & 15 & 29.4 & 19 & 37.3 & 12 & 23.5 & $<0.001$ \\
\hline no & 60 & 30.5 & 74 & 37.6 & 40 & 20.3 & 23 & 11.7 & \\
\hline Visual yes & 16 & 23.2 & 28 & 40.6 & 13 & 18.8 & 12 & 17.4 & n.s \\
\hline no & 49 & 27.4 & 67 & 34.1 & 46 & 25.7 & 23 & 12.9 & \\
\hline Sphincteric yes & 4 & 17.4 & 6 & 26.1 & 6 & 26.1 & 7 & 30.4 & n.s \\
\hline no & 61 & 27.1 & 83 & 36.9 & 53 & 23.6 & 28 & 12.4 & \\
\hline Sensory yes & 32 & 24.8 & 44 & 34.1 & 30 & 23.3 & 23 & 17.8 & n.s \\
\hline no & 33 & 27.7 & 45 & 37.8 & 29 & 24.4 & 12 & 10.1 & \\
\hline \multicolumn{10}{|c|}{$\underline{\text { Total attack duration }}^{\mathrm{e}}$} \\
\hline Short & 21 & 25.3 & 38 & 45.8 & 18 & 21.7 & 6 & 7.2 & \\
\hline Intermediate & 32 & 36.0 & 26 & 29.2 & 19 & 21.4 & 12 & 13.5 & $=0.003^{\mathrm{c}}$ \\
\hline Long & 12 & 15.8 & 25 & 32.9 & 22 & 29.0 & 17 & 22.4 & \\
\hline \multicolumn{10}{|c|}{ Therapy } \\
\hline No & 25 & 38.5 & 20 & 30.8 & 13 & 20.0 & 7 & 10.8 & \\
\hline Oral & 20 & 34.5 & 24 & 41.4 & 12 & 20.7 & 2 & 3.5 & $=0.001$ \\
\hline Intravenous & 20 & 16.0 & 45 & 36.0 & 34 & 27.2 & 26 & 20.8 & \\
\hline
\end{tabular}

${ }^{\text {a}}$ Severity was graded according to the difference between the EDSS score at the day of maximum worsening and the EDSS score before the onset of the relapse, as: very mild (0 or point), mild ( 1.5 or 2 points), moderate ( 2.5 or 3 points), and severe ( 3.5 points or more).

${ }^{b}$ Level of significance at 0.003 according to the Bonferroni correction.

${ }^{\mathrm{c}}$ Denotes the chi-square for trend test.

${ }^{\mathrm{d}}$ Here the type of FS (except mental and others) involved in each attack is cross-tabulated against grade of severity.

${ }^{\mathrm{e}}$ Calculated as the time between the date of onset of the first symptom and the date of maximum improvement of the last symptom and categorized as short ( $\leq 30$ days), intermediate (31-60 days) and long (>60 days).

The following factors were not statistically related to severity: age at onset of the attack $(<30,30-39, \geq 40)$, season of onset of the attack, infections in the preceding month, speed of onset (calculated as the time between the date of onset and the date of zenith and categorized as acute if the same day, subacute if $2-3$ days, and chronic $>3$ days), gender, age at first attack $(<30,30-39, \geq 40)$, season of birth and of first attack, oligoclonal bands at CSF examination (present/absent), Link Index $(\leq 0.70 />0.70)$ CSF IgG value $(\leq 0.40 />0.40 \mathrm{mg} / \mathrm{dl})$, total number of lesions $(0,1-8, \geq 9$ lesions), and number of gadolinium-enhancing lesions at first brain MRI $(0,1,>1$ lesions), and recovery after the first relapse (complete/incomplete). 


\section{Attack Severity}

65 attacks (26\%) were very mild, 89 (36\%) mild, 59 (24\%) moderate, and $35(14 \%)$ severe. Table 1 shows the degree of severity according to the attack-related predictive factors. Pyramidal and brainstem FS, number of FS involved and total attack duration were related to severity, the last two with a clear trend. As expected, iv. therapy was most frequent in more severe attacks. The highest risk of a severe attack was for pyramidal (OR 3.5, 95\% CI 1.7-7.5) and cerebellar FS (3.5, 1.5-8.2), followed by sphincteric (3.1, 1.2$8.1)$ and brainstem (2.3, 1.1-5.1). Sensory (1.9, 0.9-4.1) and visual (1.4, 0.7-3.1) FS did not significantly increase the risk of a severe attack. Attacks with visual symptoms were more frequently monosymptomatic $(45 / 69,65 \%)$ than those with sensory $(50 / 129,39 \%)$, brainstem $(17 / 51,33 \%)$, motor $(31 / 98,32 \%)$, cerebellar $(5 / 32,16 \%)$, and sphincteric symptoms $(1 / 23,4 \%)$. To avoid bias determined by the multiple inclusion of attacks from single patient, we correlated patient-related predictive factors to attack severity separately for the first and second attack: none of them was correlated with the degree of severity (data not shown). In the multivariate model, the number of symptoms (mono vs. bipolysymptomatic) was the only variable that explained severity (OR for severe attacks: 6.6; 95\% CI 2.9-15.3). Since the number of FS is highly related with severity due to the measurement procedure, we performed another analysis, without number of FS. The following variables were retained after stepwise selection: pyramidal (OR 3.6, 1.7-7.7), brainstem $(2.6,1.2-6.0)$, and long duration $(2.5,1.2-5.3)$.

\section{Attack Duration}

83 attacks (34\%) were classed as short, $89(36 \%)$ as intermediate, and $76(31 \%)$ as long. Table 2 shows the duration according to the attack-related predictive factors. Number of FS, type of FS involved (sphincteric and sensory) and severity of the attack were related to duration. The risk of a long duration was significant only for sphincteric $(5.0 ; 2.0$ $12.5)$, and sensory $(2.1,1.2-3.6)$ FS involvement. None of patient-related predictive factors was correlated with the duration of both the first and second attack (data not shown).

Table 2. Duration of 248 MS Attacks, According to Attack-Related Characteristics

\begin{tabular}{|c|c|c|c|c|c|c|c|}
\hline \multirow[b]{3}{*}{ Predictive Factor } & \multicolumn{4}{|c|}{ Attack duration ${ }^{a}$} & & & \multirow{3}{*}{ p-value } \\
\hline & \multicolumn{2}{|c|}{ short } & \multicolumn{2}{|c|}{ intermediate } & \multicolumn{2}{|c|}{ long } & \\
\hline & $\mathbf{N}$ & $\%$ & $\mathbf{N}$ & $\%$ & $\mathbf{N}$ & $\%$ & \\
\hline \multicolumn{8}{|c|}{ Number of FSs involved } \\
\hline Monosymptomatic & 59 & 39.6 & 53 & 35.6 & 37 & 24.8 & \\
\hline Bisymptomatic & 17 & 29.8 & 25 & 43.9 & 15 & 26.3 & $<0.0005^{\mathrm{c}}$ \\
\hline Polysymptomatic & 7 & 16.7 & 11 & 26.2 & 24 & 57.1 & \\
\hline \multicolumn{8}{|c|}{$\underline{\text { Type of FS involved }}^{\mathrm{d}}$} \\
\hline Pyramidal yes & 30 & 30.6 & 35 & 35.7 & 33 & 33.7 & n.s. \\
\hline no & 53 & 35.3 & 54 & 36.0 & 43 & 28.7 & \\
\hline Cerebellar yes & 10 & 31.2 & 9 & 28.1 & 13 & 40.6 & n.s. \\
\hline no & 73 & 33.8 & 80 & 37.0 & 63 & 29.2 & \\
\hline Brainstem yes & 18 & 35.3 & 19 & 37.3 & 14 & 27.5 & n.s. \\
\hline no & 65 & 33.0 & 70 & 35.5 & 64 & 31.5 & \\
\hline Visual yes & 23 & 33.3 & 23 & 33.3 & 23 & 33.3 & n.s. \\
\hline no & 60 & 33.5 & 66 & 36.9 & 53 & 29.6 & \\
\hline Sphincteric yes & 2 & 8.7 & 6 & 26.1 & 15 & 65.2 & $<0.001$ \\
\hline no & 81 & 36.0 & 83 & 36.9 & 61 & 27.1 & \\
\hline Sensory yes & 31 & 24.0 & 49 & 38.0 & 49 & 38.0 & $=0.002$ \\
\hline no & 52 & 43.7 & 40 & 33.6 & 27 & 22.7 & \\
\hline \multicolumn{8}{|c|}{ Severity } \\
\hline Very mild & 21 & 32.3 & 32 & 49.2 & 12 & 18.5 & \\
\hline Mild & 38 & 42.7 & 26 & 29.2 & 25 & 28.1 & \\
\hline Moderate & 18 & 30.5 & 19 & 32.2 & 22 & 37.3 & $=0.003^{\mathrm{c}}$ \\
\hline Severe & 6 & 17.1 & 12 & 34.3 & 17 & 48.6 & \\
\hline
\end{tabular}

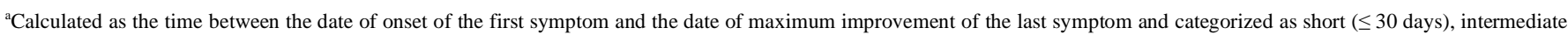
(31-60 days) and long (>60 days).

See Table 1 for the other definitions

The following factors were not statistically related to duration: attack therapy, age and season of onset of the attack, infections in the preceding month, speed of onset, gender, age at first attack, season of birth and of first attack, oligoclonal bands at CSF examination, Link Index, CSF IgG, total number of lesions and number of gadolinium-enhancing lesions at first brain MRI, and recovery after the first relapse (see table 1 for definitions). 
In the multivariate model, sphincteric $(4.4,1.7-11.0)$ and sensory symptoms $(1.8,1.0-3.2)$ were the only variables explaining duration.

\section{Probability of a Severe or Long Second Attack}

In a different approach, we tested whether the probability of a second severe or long attack was influenced by severity and duration of the first. Since the number of second severe attacks was small $(\mathrm{N}=7)$ we grouped them with moderate second attacks $(\mathrm{N}=13)$. The probability of a second moderate/severe or long attack was not influenced by severity or duration of the first (Figs. 1 and 2). The 3-year probability of a second moderate/severe attack was $21 \%$ (4-38) for a patients with a severe first attack and 22\% (12-32) for those with a very mild-mild-moderate first attack $(\mathrm{p}=0.71)$. It was $23 \%(9-37)$ for a patients with a long first attack, and $21 \%$ (10-32) for those with a short-intermediate first attack $(\mathrm{p}=0.89)$. The 3 -year probability of a second long attack was

Probability of a second moderate/severe attack according to severity of the first

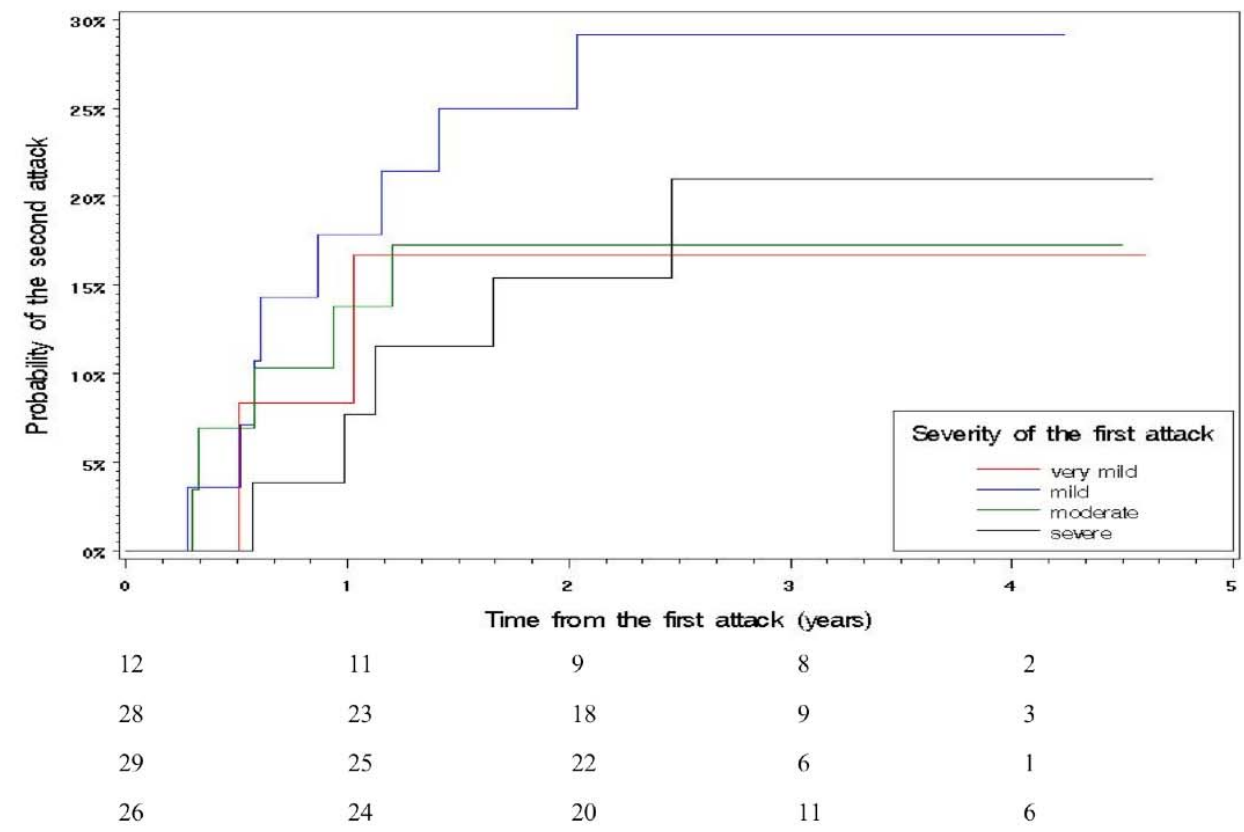

No. at risk

Very mild

Mild

Moderate

Severe

24

Probability of a second moderate/severe attack according to duration of the first

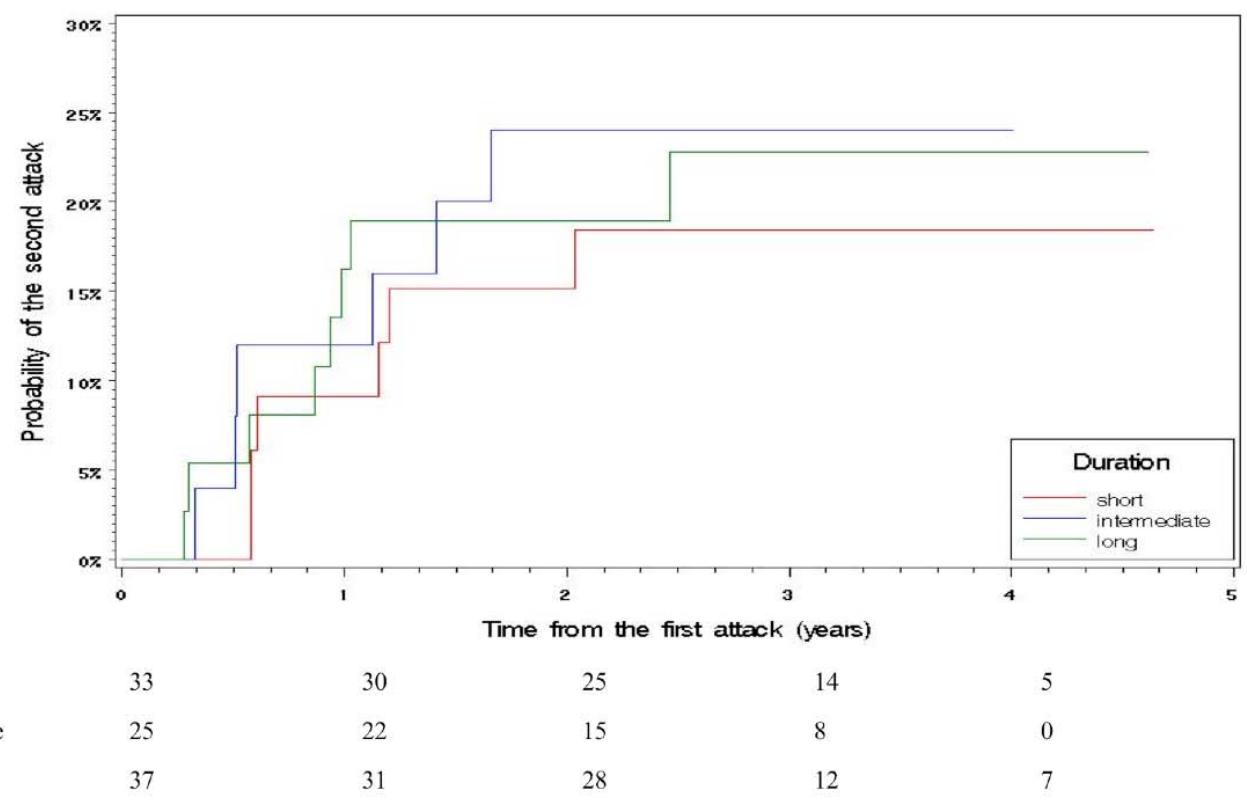

Fig. (1). Cumulative time-dependent probability of a second moderate/severe attack according to severity and duration of the first. The 3year probabilities were: $21 \%$ (4-38) for patients with a severe first attack, $17 \%$ (4-31) for moderate, 29\% (12-46) for mild, and 17\% (0-38) for very mild $(\mathrm{p}=0.70) ; 23 \%(9-37)$ for patients with a long first attack, $24 \%(7-41)$ for intermediate and $18 \%(5-32)$ for short ( $\mathrm{p}=0.81)$. 
Probability of a second long attack according to severity of the first

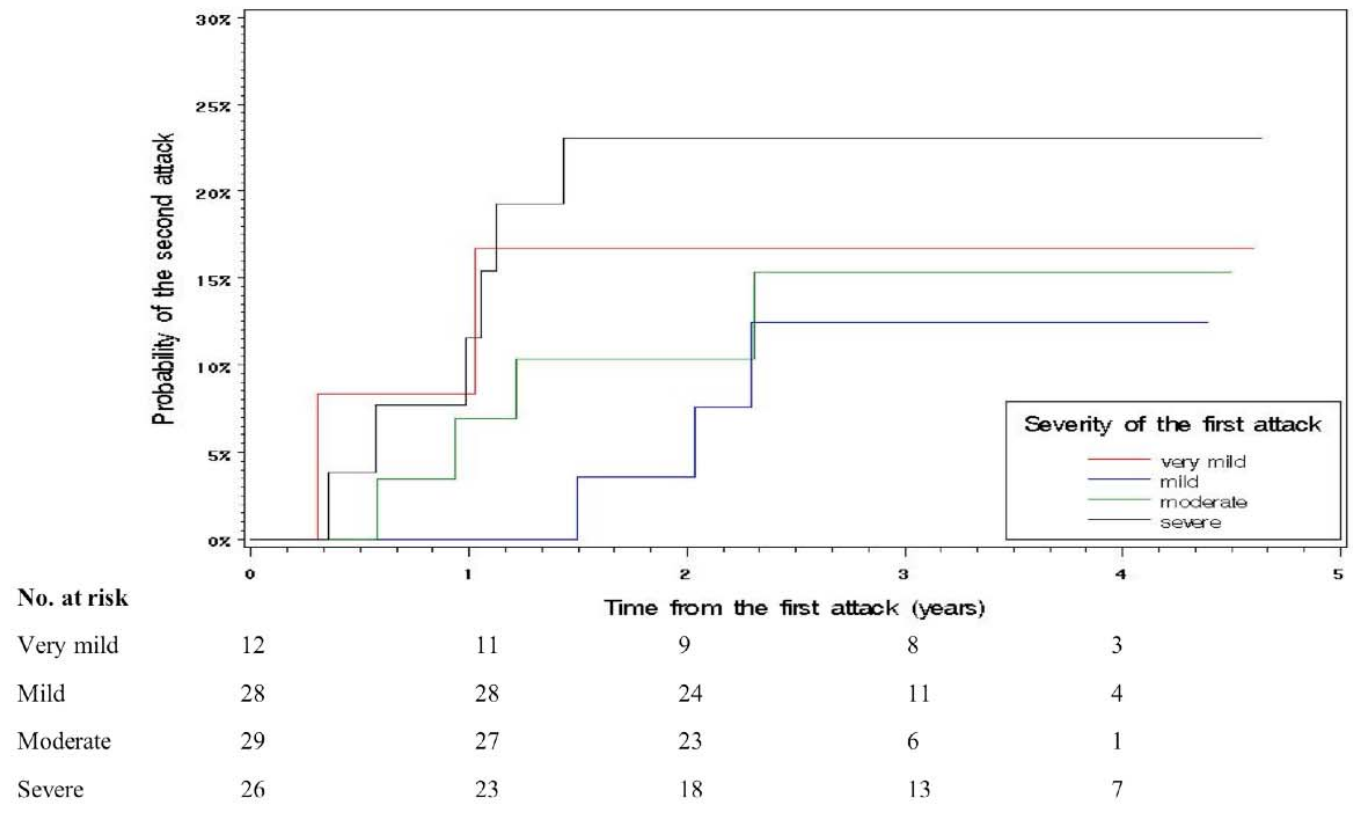

Probability of a second long attack according to duration of the first

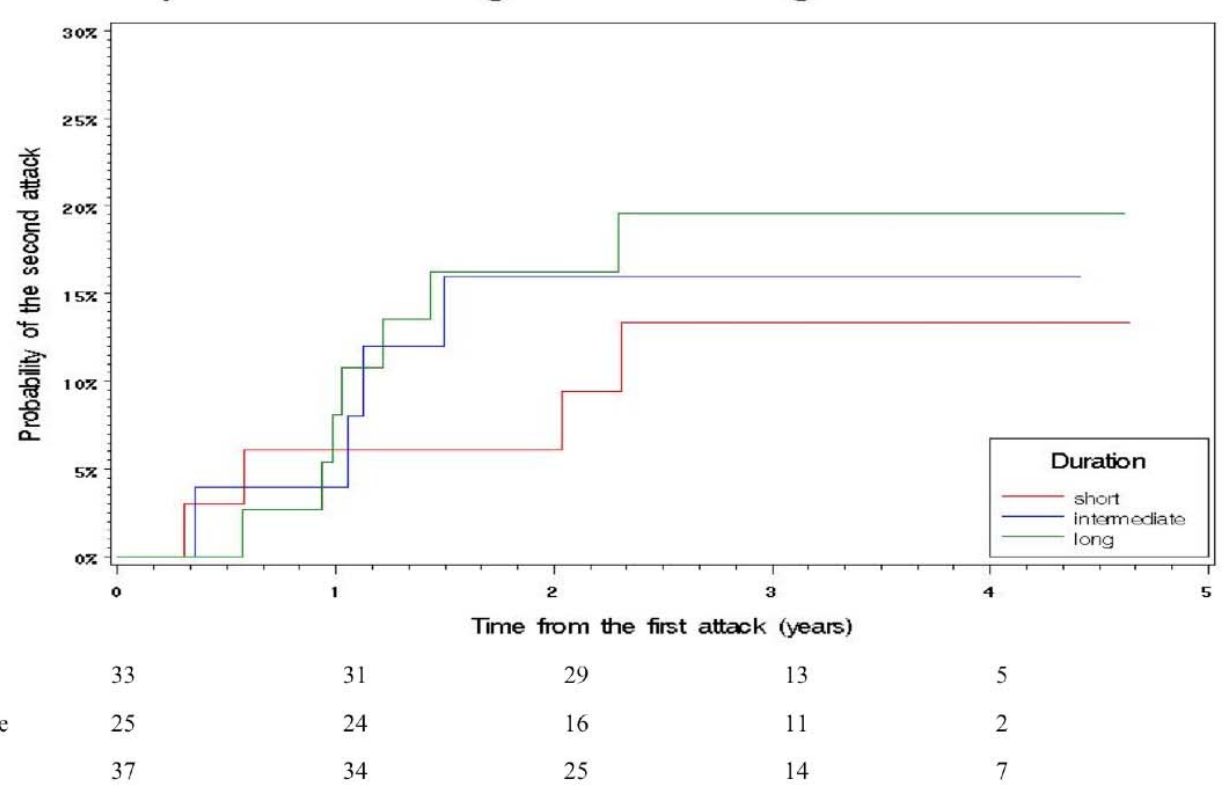

Fig. (2). Cumulative time-dependent probability of a second long attack according to severity and duration of the first. The 3-year probabilities were: $23 \%$ (7-39) for patients with a severe first attack, $15 \%(1-29)$ for moderate, $13 \%$ (0-26) for mild, and $17 \%(0-38)$ for very mild $(\mathrm{p}=0.61) ; 20 \%(7-33)$ for patients with a long first attack, $16 \%(2-31)$ for intermediate and $13 \%(1-26)$ for short $(\mathrm{p}=0.75)$.

$23 \%$ (7-39) for a patients with a severe first attack, and $14 \%$ (6-23) for those with a very mild-mild-moderate first attack $(\mathrm{p}=0.22)$. It was $15 \%(5-24)$ for patients with a long first attack, and 20\% (7-32) for those with a short-intermediate first attack $(\mathrm{p}=0.53)$. We performed a multivariate analysis, including all attack-related factors, gender and recovery after the first attack (Table 3); other factors not significant in the univariate analysis were not included. The only predictive (negative) variable for a moderate/severe second attack was incomplete recovery from the first attack.

\section{DISCUSSION}

In accordance with our previous study [1], we divided predictive factors into attack-related and patient-related. None of the patient-related features was predictive of attacks severity or duration. Among attack-related factors, the number of FS involved was the only predictor of severity after the multivariate analysis; since the grade of severity in our categorization was not independent from the number of affected FS, we cannot draw any conclusion on the relation between the spread of the damage in each attack and its se- 
Table 3. Probability of a Second Moderate/Severe or Long Attack: Multivariate Analysis (N=95)

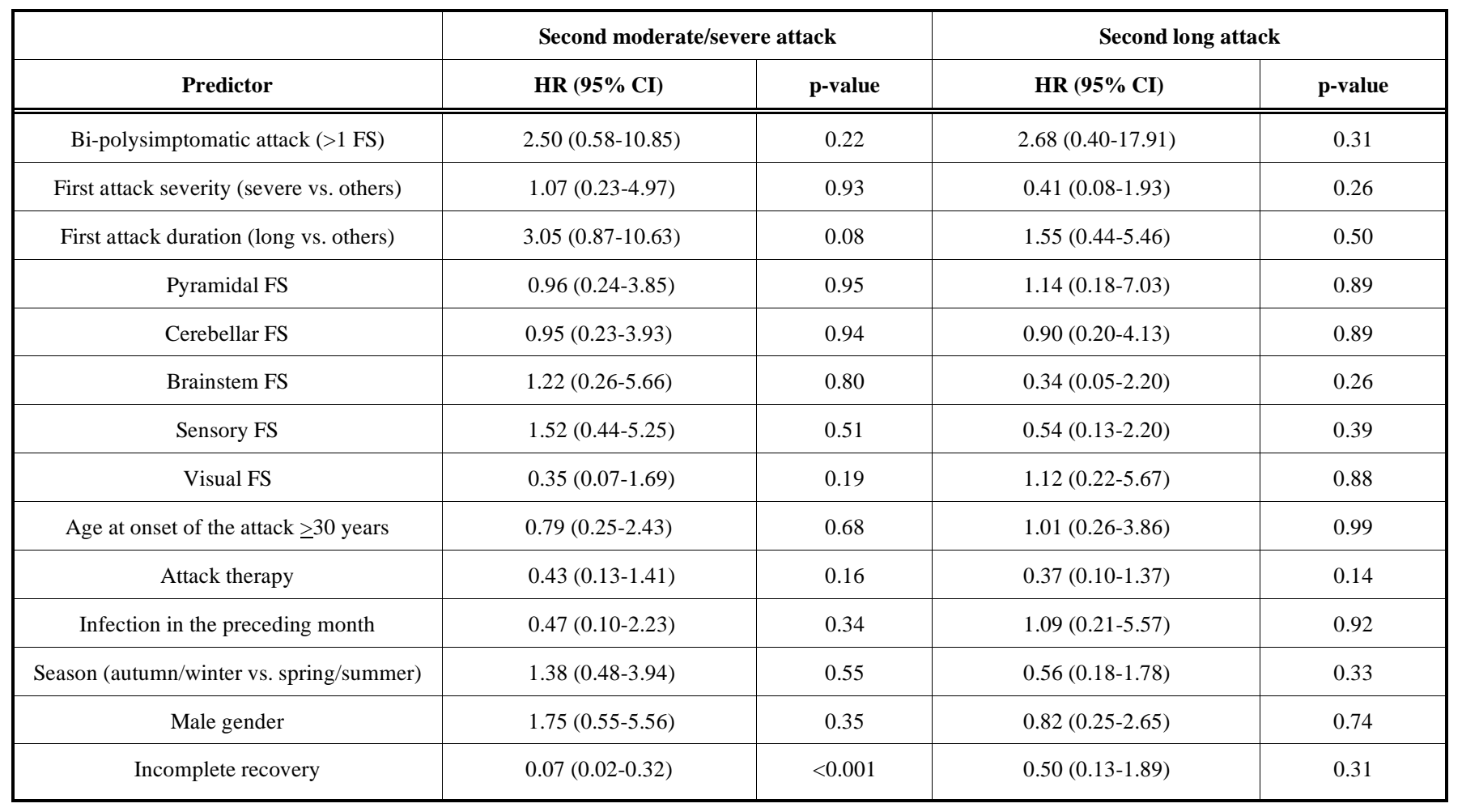

verity. Severity clearly differed among FS, pyramidal and brainstem being the most severe both in the univariate and the multivariate analyses. Similar differences were found by Mowry et al., [4] in 330 patients. Whether this has biological plausibility (e.g. a different degree of inflammation in different FS) or is a mere consequence of the score system is not clear. Score levels of each FS are not equivalent, and moving from one score to another might not have the same biological meaning for visual, sensory or pyramidal FS. The lower severity of attacks with optic nerve involvement may also depend on the fact that they were more frequent monosymptomatic. Attack duration was one of the factors determining severity; a longer exposure to toxic substances [16] might determine more damage and lower possibility of recovery.

Assessment of duration is hampered by uncertainly as to the exact moment when an attack ends. Despite the fact that no mean duration could be established since attack ends had to be estimated, our three categories clearly identify different durations. Severity and number of symptoms were predictive of long duration in the univariate analysis, but the multivariate model retained only two individual FS, sphincteric and sensory. Attacks with sphincteric symptoms were almost always polysimptomatic and it is also likely that the end of such attacks cannot be readily evaluated. We couldn't compare these data, because factors predicting duration have never been investigated hitherto.

Mowry et al., [4] claimed a substantially increased risk of a more severe subsequent event for those with poor recovery from the first, and a tendency to repeat the degree of severity in attacks subsequent to the first. We did not find any relation of severity or duration in the first attack to severity or duration in the second. However the grading systems were different and cannot be fully compared. Patients with incom- plete recovery from the first attack had a lower probability to have a moderate/severe, but not a long second attack. This should be interpreted in the context of our measure procedure: patients with a residual high score from the first attack were probably unable to reach a higher score for the second, especially if this occurred in the same FS. Thus attacks with equal severity scored higher and were deemed longer when the first recovered to EDSS 0. Evaluation of third and later attacks could help to understand this point better, but we had a too low number of such attacks to analyze.

Some limitations of our study must be duly acknowledged. Firstly, our analyses was confined to attacks during early stages of the natural history of MS, and our findings cannot be automatically transferred to later attacks. Secondly, steroid management was not standardized, and treatment was left to the patient's own physician. Patients with more severe attacks were treated more frequently with steroids. Since many attacks were not immediately treated, the physicians were probably able to establish their severity and treat them accordingly. Thirdly, we used only EDSS to score variations during attacks. This had the limit of lower responsiveness than other scales or impossibility to detect cognitive symptoms, but assured good agreement between the attack evaluators and practicability [1]. Fourthly, one third of our attacks were anamnestic. Although every effort was made to collect as much information as possible, we cannot entirely rule out a bias due to the diversity of data collection. However, inclusion of both anamnestic and observed attacks allowed examination of a consecutive series of events, and separate analysis of anamnestic and observed attacks identified the same predictors of severity and duration in both groups. Fifthly, since our study is hospital-based, a selection bias towards more severe cases cannot be excluded; how- 
ever, most MS patients from our catchment areas are seen soon after the onset of symptoms and rarely refer to other hospitals for an initial assessment.

In conclusion, our data give support to the idea that each attack has its own severity and duration dependent on some attack-related predictive factors, but independent on individual-related predictors or of what happened in previous attacks [1]. Severity displays an intriguing relation with recovery in many studies $[1,2,4]$. However, if a severe attack (greater inflammation) is a risk factor for an incomplete recovery (failed remyelination), the reverse process has no evident foundation, since repairing difficulties in one location are not necessarily associated with subsequent greater inflammation in the same or other locations. However, we did not consider other variables that may serve to predict attack characteristics, such as the genetic background, for example HLA. An analysis of genetic predictors is warranted.

\section{REFERENCES}

[1] Leone MA, Bonissoni S, Collimedaglia L, et al. Factors predicting incomplete recovery from relapses in multiple sclerosis: a prospective study. Mult Scler 2007; 14: 485-93.

[2] West T, Wyatt M, High A, et al. Are initial demyelinating event recovery and time to second event under differential control? Neurology 2006; 67: 809-13.

[3] Vercellino M, Romagnolo A, Mattioda A, et al. Multiple sclerosis relapses: a multivariable analysis of residual disability determinants. Acta Neurol Scand 2009; 119: 126-30.

[4] Mowry EM, Pesic M, Grimes B, et al. Demyelinating events in early multiple sclerosis have inherent severity and recovery. Neurology 2009; 72: 602-8.

[5] Scott TF, Schramke CJ. Poor recovery after the first two attacks of multiple sclerosis is associated with poor outcome five years later. J Neurol Sci 2010; 15: 292(1-2): 52-6.
[6] Ramsaransing GS, De Keyser J. Predictive value of clinical characteristics for 'benign' multiple sclerosis. Eur J Neurol 2007; 14: 8859.

[7] Langer-Gould A, Popat RA, Huang SM, et al. Clinical and demographic predictors of long-term disability in patients with relapsingremitting multiple sclerosis: a systematic review. Arch Neurol 2006; 63: 1686-91.

[8] Hirst C, Ingram G, Pearson O, Pickersgill T, Scolding N, Robertson N. Contribution of relapses to disability in multiple sclerosis. J Neurol 2008; 255: 280-7.

[9] Miller D, Barkhof F, Montalban X, et al. Clinically isolated syndromes suggestive of multiple sclerosis, part I: natural history, pathogenesis, diagnosis, and prognosis. Lancet Neurol 2005; 4: 281-8.

[10] McDonald WI, Compston A, Edan G, et al. Recommended diagnostic criteria for multiple sclerosis: guidelines from the International Panel on the diagnosis of multiple sclerosis. Ann Neurol 2001; 50: 121-7.

[11] Kurtzke JF. Rating neurologic impairment in multiple sclerosis: an expanded disability status scale (EDSS). Neurology 1983; 33: 1444-52.

[12] Liu C, Blumhardt LD. Assessing relapses in treatment trials of relapsing and remitting multiple sclerosis: can we do better? Mult Scler 1999; 5: 22-8.

[13] Schumacker GA, Beebe G, Kibler RF, et al. Problems of experimental trials of therapy in multiple sclerosis: report by the panel on the evaluation of experimental trials of therapy in multiple sclerosis. Ann N Y Acad Sci 1965; 122: 552-68.

[14] Trojano M, Avolio C, Manzari C, et al. Multivariate analysis of predictive factors of multiple sclerosis course with a validated method to assess clinical events. J Neurol Neurosurg Psychiatr 1995; 58: 300-306.

[15] Sas Institute Inc. SUGI supplemental library user's guide. Version $8^{\text {th }}$ ed. Cary, NC: SAS Institute, 1999-2001.

[16] Smith KJ, McDonald WI. The pathophysiology of multiple sclerosis: the mechanisms underlying the production of symptoms and the natural history of the disease. Philos Trans R Soc Lond B Biol Sci 1999; 354: 1649-73.

(C) Naldi et al.; Licensee Bentham Open.

This is an open access article licensed under the terms of the Creative Commons Attribution Non-Commercial License (http://creativecommons.org/licenses/by-nc/3.0/) which permits unrestricted, non-commercial use, distribution and reproduction in any medium, provided the work is properly cited. 